\title{
Biflmaging
}

\section{Channel Surfing: Creating Different Color Combinations from Multiple-Label Images}

\author{
Steve Paddock \\ Howard Hughes Medical Institute, University \\ of Wisconsin, Madison, WI, USA
}

Mapping the distribution of more than one macromolecule in a tissue is now a relatively routine technique that uses multiple-label fluorescence $(2,7)$ and one of several different digital imaging methods $(9,10)$, including wide-field, confocal, deconvolution, or multiple-photon microscopy. Methods of specimen preparation continue to be refined, especially as new fluorescent probes are developed and become more widely available (6)

A convenient method for displaying three grayscale images collected from the same region of a triple-labeled specimen uses the channels feature of the popular image manipulation program, Adobe ${ }^{\circledR}$ Photoshop ${ }^{\circledR}$. Here, the three grayscale im ages are pasted into the red, green, and blue channels of a $\mathrm{red} / \mathrm{green} / \mathrm{blue}$ (RGB) image where they appear as a three-color merged image $(7,8)$. For the method to be successful, the grayscale images should be collected from precisely the same region of the specimen so that they are in register with one another. Some shifting of the images relative to each other can be achieved in Photoshop to bring them back into register. It is preferable, however, to use both a microscope that collects three images at three different excitation wavelengths simultaneously and high-quality objective lenses (2).

To glean the most information from combining the three grayscale images, one may resort to "channel surfing" using Photoshop. Here, grayscale images are simply rearranged within the channels of Photoshop to produce images with different color combinations. Images are subsequently viewed and printed using a digital color printer. They can then be compared, and the best one chosen that satisfies the criteria of scientific information along with the all-important aesthetic appeal.

For our example of channel surfing, I have chosen a confocal image of a triple-labeled third instar Drosophila haltere imaginal disc. The imaginal discs are made up of pouches of cells at the time of larval hatching and are destined to form appendages such as the legs, the wings, the halteres, the genitalia, and the eyes. The halteres are the balance organs of the fly, located behind the wings, and are related to the wings by their development and their evolution $(12,13)$. At the third instar stage of development, the imaginal discs are relatively flat epithelial sheets of cells. They are currently the subjects of much genetic and molecular dissection, which has greatly benefited from the improved resolution and image registration afforded by confocal imaging of multiply labeled specimens. The spatial distribution of up to three macromolecules has been mapped during development using this approach $(3,8,12)$.

Our specimen has been labeled with antibodies to three proteins using secondary antibodies labeled with rhodamine, fluorescein, and cyanine 5 (3) and imaged using a confocal laser-scanning microscope (Bio-Rad ${ }^{\circledR}$ MRC1024; Bio-Rad
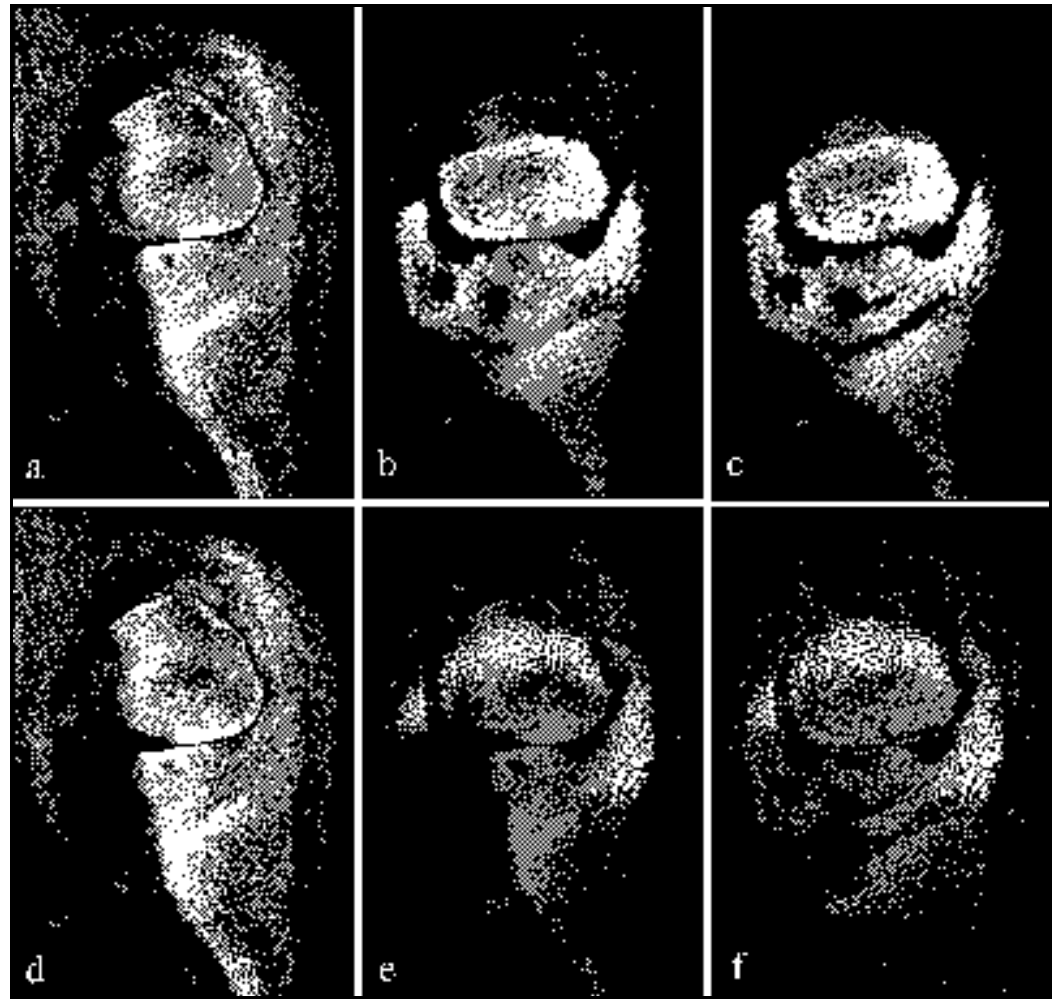

Figure 1. Triple-labeled Drosophila third instar haltere imaginal disc. The six different color combinations of red, green, and blue are displayed using channel surfing in PhotoShop. Note that extra colors appear in the images in regions of overlap, such that the regions of purple in (a) and (d) are the result of signal in the same regions (nuclei) of the red and the blue channels. Note also that the single blue colors do not reproduce well especially in (c) and (f). For our purposes, the color combinations in (a), (d), or (e) would convey the most information. (Specimen courtesy of Jim Williams). 
Laboratories, Hercules, CA, USA). The three-color image was subsequently transferred to Photoshop (5) where the channels were rearranged by copying and pasting grayscale images into a second blank RGB image of the same size and pixel resolution as the source image (Figure 1).

Channel surfing can be used to rearrange the colors in doubly labeled images, too (Figures 2 and 3). These images consist of two grayscale images in two separate channels with a black image in the third channel. They were produced from the triple-labeled image in Figure 1 by pasting a black image into one of the channels. Any overlap of the images appears as an additive color change, such that regions of red that overlap with green appear as yellow (Figure 2a). Here, the images can be simply rearranged in the channels to give either a red/blue (Figure 2b) or a blue/green image (Figure 2c). Single blue colors should generally be avoided because they lack contrast in a final digital print (Figure 2, b and c). More colors can be produced by pasting the same image into two channels; for exam ple, a green/purple image is produced by pasting the same grayscale image into the red and the blue channels with the second image pasted into the green channel (Figure 2d). Additional combinations include light blue/red (Figure 2e) and blue/yellow (Figure 2f). Here, regions of overlap will appear white because there is an additive contribution from all three channels (Figure 2, d, e, and f). Improved blue can be achieved by pasting images into two channels in this way (Figure 2e).

In addition to displaying the distribution of different macromolecules in a triple-labeled sample, channel surfing can be used to display depth information within a specimen $(5,8)$. A Z-series of images is collected through the specimen, for example, using a confocal microscope to preserve the $\mathrm{X}, \mathrm{Y}$, and $Z$ registration between all of the images in the $Z$-series file. Three images can be chosen from the stack of images (or three groups of images from the upper, middle, and lower regions of

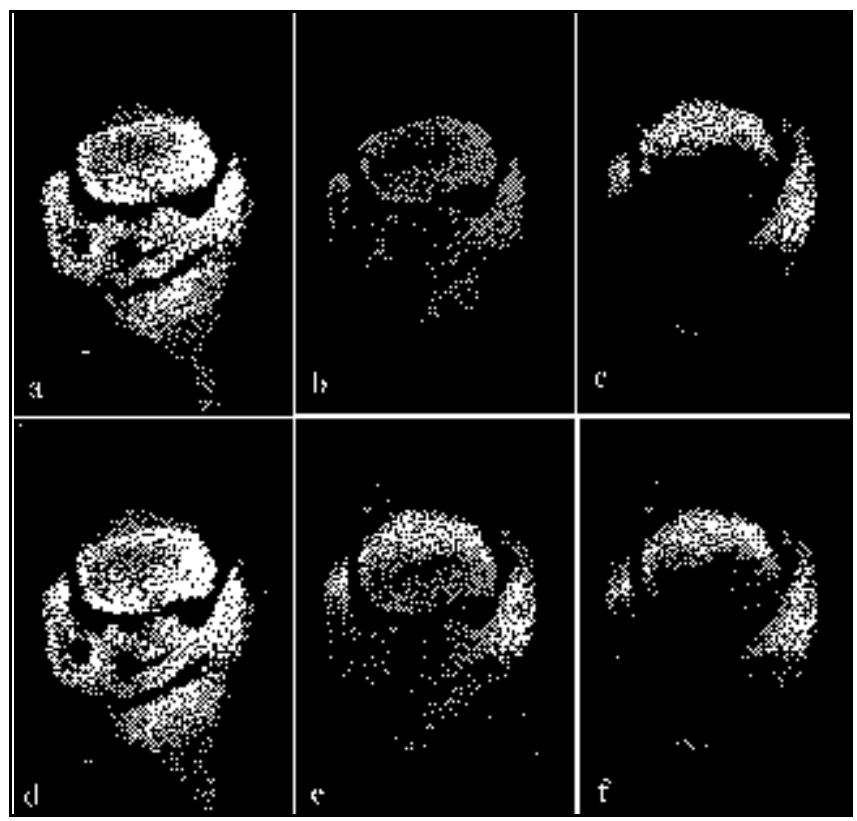

Figure 2. Channel surfing in a doubly labeled image with regions of overlap. Both of these antibody probes are located in the nucleus. the Z-series file can be merged into three separate grayscale images) and pasted into the red, green, and blue channels of Photoshop so that the colors red, green, and blue are assigned to structures at different depths in the specimen (Figure 4).

In a similar way, single grayscale images of three different time points can be extracted from a time-lapse movie file, pasted into the RGB channels, and merged into a single RGB color image so that color is now mapped to time to display changes in the position of a particular structure in a single image.

The combination of colors in a multiply labeled image is clearly important for efficiently communicating the biological information nested there. By channel surfing, it is a relatively simple task to explore these combinations. Because of the exquisite specificity of immunofluorescence, many of the images are rather abstract when viewed in a single channel alone and can only really be interpreted when a second or third image of a known landmark within the tissue is displayed at the same time.

This is especially true of images of fluorescent in situ hybridization (FISH) of chromosome spreads or nuclei, which are often made up of several bright dots on a mostly black background, and the images can only be interpreted when a second, counterstained image of the entire chromosome or nucleus is collected and merged with the brightly stained centers of hybridization $(1,11)$. The dimeric nucleic acid dyes such as YOYO-1 or TOTO-3 are excellent counterstains for these purposes. In addition, wheat germ agglutinin can be used to stain the nuclear periphery rather than most of the nucleus (14).

Additional probes are available that label landmarks within tissues. Fluorescently labeled phalloidin is a convenient probe to include in a triple labeling strategy for imaging cell outlines (4). An extra landmark can be gained by including two dyes with similar emission colors in the sample, such as a nuclear dye like TOPRO and cyanine 5-labeled phalloidin, so that the cell outlines and nuclei appear in the same channel. In this way, a macromolecule can be traced to a specific cellular compartment: to the outside of the cell, to the cytoplasm, or to the nucleus. Moreover, additional fluorescent dyes are available that would further narrow the macromolecule's location in the cytoplasm, for example, to either the mitochondria, the Golgi, the endoplasmic reticulum, or to elements of the cytoskeleton (6). The appearance of specific color combinations in images of these multiple-labeled specimens is useful for mapping macromolecules to cellular compartments.

The display of multiply labeled images using RGB colors in Photoshop is especially useful for combinations of red-, green-, and blue-emitting dyes (rhodamine, fluorescein, and Hoechst 3347) in which the emission colors are matched to the red, green, and blue channels, respectively. This means that the distribution of colors faithfully reproduces in the digital image what would be seen by eye using an epifluorescence microscope. However, in many cases, the emission colors of the fluorochromes used in a protocol do not fit the RGB map. To illustrate, cyanine 5 is a popular fluorochrome that emits in the far red. This wavelength is difficult to visualize by eye, appearing very dark red in well-stained samples, but well within the detection range of most digital imaging devices. Here, red, green, or blue could be assigned to the cyanine 5 channel.

Even when the colors match the RGB map, the resulting merged image may not adequately convey the biological in- 


\section{Biermaging}

formation contained in the image. This may be a question of the aesthetics of different color combinations combined with technical aspects of reproducing certain colors using digital printing devices. For example, it is often difficult to reproduce the contrast of single blues in digital prints. Combinations of red and green may also be a problem for people affected with color blindness, and it may be necessary to change the color combinations for them to clearly visualize the data. Above all, it is essential to choose the color combinations and probes so that there is not excessive overlap, which can lead to highly colored and confusing images. The ultimate goal of channel surfing is to produce an image that conveys the biological information in the clearest way possible and is also aesthetically pleasing to the eye.

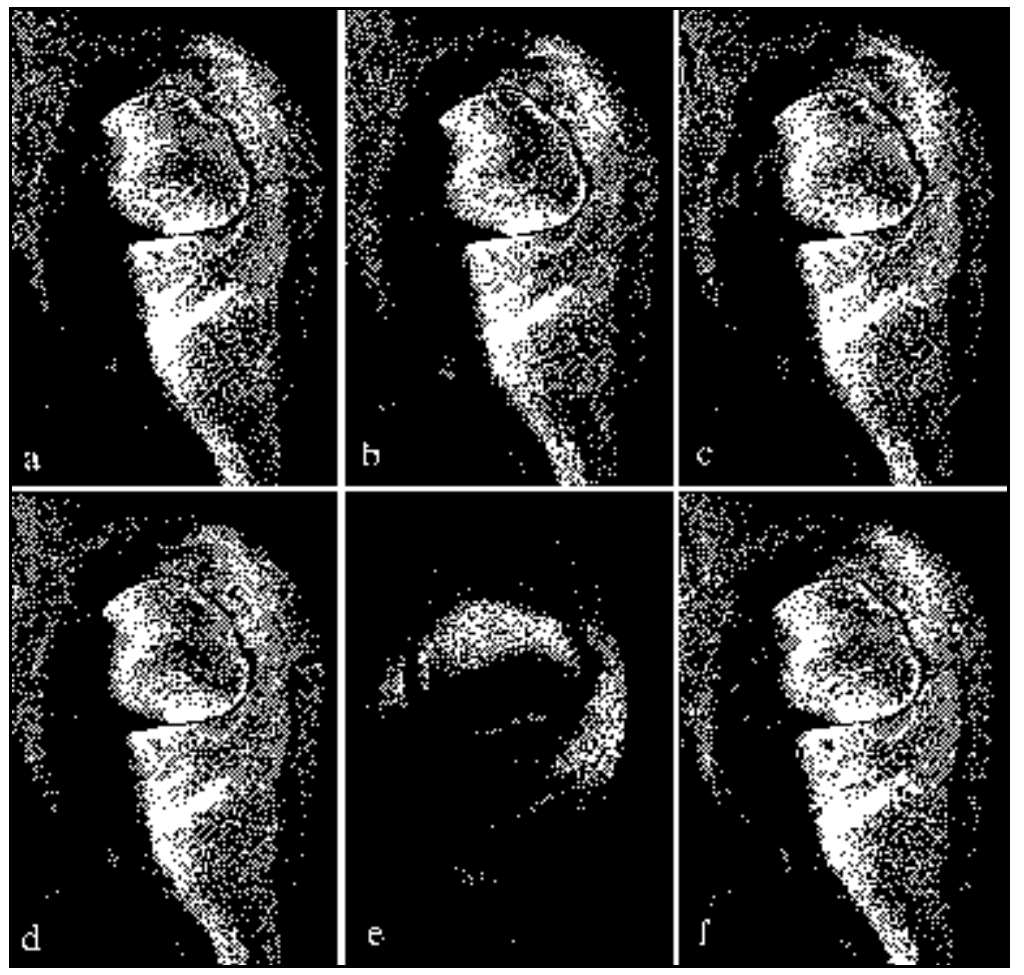

Figure 3. Channel surfing in a doubly labeled image with no overlapping of signal. Here, one of the antibody probes labels nuclei [red staining in (a)] and the second one labels an extracellular protein [green in (a)]. Some yellow is present because this is a relatively low magnification image with low pixel resolution. A higher magnification image of higher pixel resolution confirmed the lack of overlap of expression.

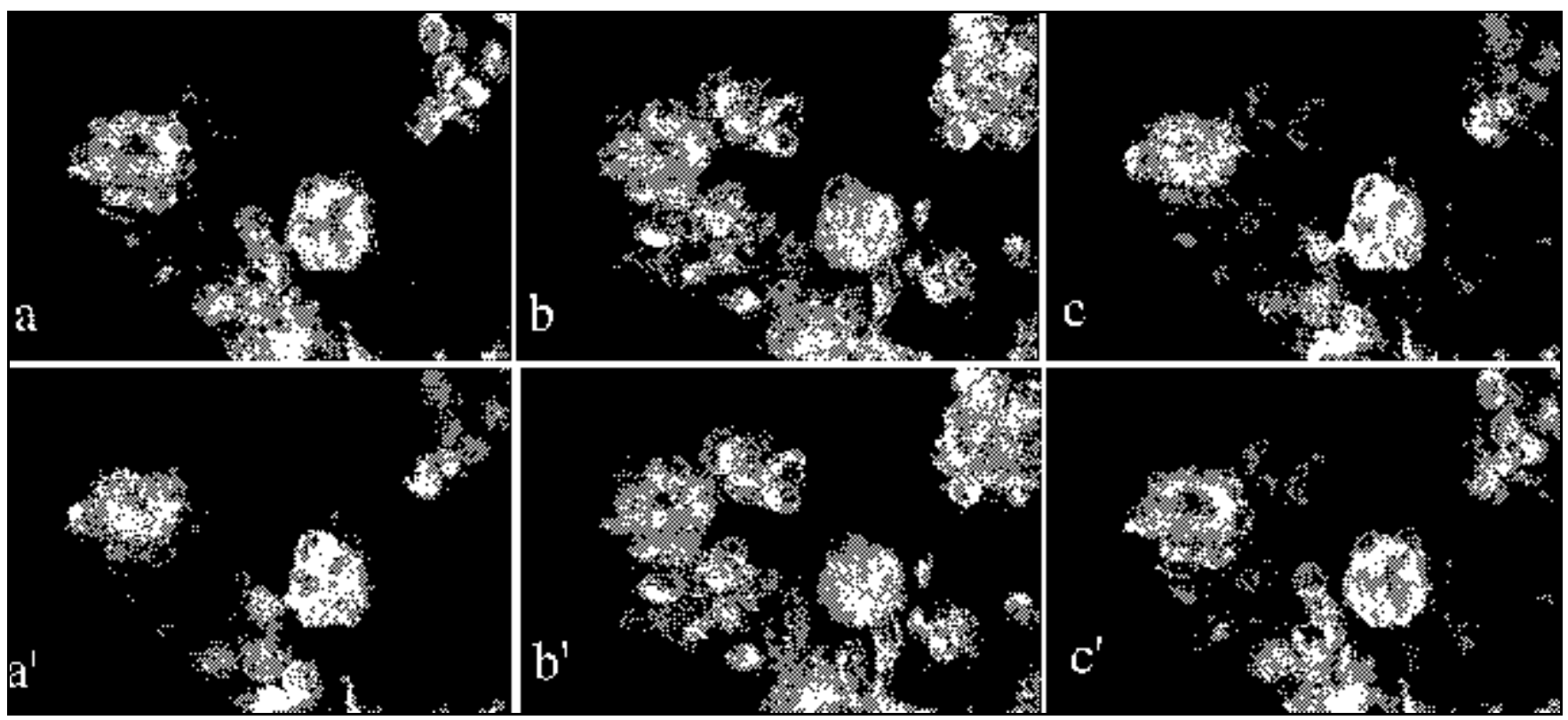

Figure 4. Channel surfing in Z-series-color coding to depth. Rat BN/MSV cells form three-dimensional aggregates in culture, which can be distinguished as multicolored regions from single cells growing on the substratum viewed as single blue in $\left(\mathrm{a}, \mathrm{a}^{\prime}\right)$, single green in $\left(\mathrm{b}, \mathrm{b}^{\prime}\right)$, or single red in (c, $\left.\mathrm{c}^{\prime}\right)$. Here, the poor contrast of single blue may be an advantage for resolving the highly colored cell aggregates. (Specimen courtesy of Tim Hammond, Tulane University). 


\section{REFERENCES}

1.Albertson, D.G., R.M. Fishpool, and P.S. Birchall. 1995. Fluorescence in situ hybridization for the detection of DNA and RNA. Methods Cell Biol. 48:339-364.

2.Brelje, T.C., M.W. Wessendorf, and R.L. Sorenson. 1993. Multicolor laser scanning confocal immunofluorescence microscopy: practical applications and limitations. Methods Cell Biol. 38:98-177.

3.Brown, N.L. 1998. Imaging gene expression using antibody probes. Methods Mol. Biol. 122:75-91.

4.Condic, M.L., D. Fristrom, and J.W. Fristrom. 1991. Apical cell shape changes during Drosophila imaginal leg disc elongation: a novel morphogenetic mechanism. Development 111:23-33.

5.Halder, G. and S.W. Paddock. 1998. Presentation of confocal images. Methods Mol. Biol. 122:373-384.

6.Haugland, R.P. 1999. Handbook of Fluorescent Probes and Research Chemicals, 7th ed. Molecular Probes Inc., Eugene, OR.

7.Paddock, S.W., J.A. Langeland, P.J. DeVries, and S.B. Carroll. 1993. Three-color immunofluorescence imaging of Drosophila embryos by laser scanning confocal microscopy. BioTechniques 14:42-48.

8.Paddock, S.W., E.J. Hazen, and P.J. DeVries. 1997. Methods and applications of three colour confocal imaging. BioTechniques 22:120-126.

9.Paddock, S.W. 1999. Confocal laser scanning microscopy - an overview. BioTechniques 27:992-1004

10.Pawley, J.B. 1995. Handbook Of Biological Confocal Microscopy, 2nd ed. Plenum Press, New York.

11.Schrock, E.S., T. du Manoir, B. Veldman, J. Schoell, M.A. Wienberg, Y. Ferguson-Smith, D.H. Ning, I. Ledbetter et al. 1996. Multicolor spectral karyotyping of human chromosomes. Science 273:494-497.

12. Weatherbee, S., G. Halder, A. Hudson, J. Kim, and S. Carroll. 1998. Ultrabithorax regulates genes at several levels of the wing-patterning hierarchy to shape the development of the Drosophila haltere. Genes Dev. 10:1474-1482.

13. Weatherbee, S., F. Nijhout, L. Grunert, G. Halder, R. Galant, J. Selegue, and S. Carroll. 1999. Ultrabithorax function in butterfly wings and the evolution of insect wing patterns. Curr. Biol. 9:109-115.

14. Wilkie, G.S., A.W. Shermoen, P.H. O'Farrell, and I. Davis. 1999. Transcribed genes are localized according to chromosomal position within polarized Drosophila embryonic nuclei. Curr. Biol. 9:1263-1266.

Address correspondence to Dr. Steve Paddock, Howard Hughes Medical Institute, Dept. Molecular Biology, University of Wisconsin, 1525 Linden Drive, Madison, WI 53706, USA. e-mail: paddock @facstaff.wisc.edu

Suggestions for contributions to the Biolmaging feature are welcomed by its editor, Dr. Steve Paddock (paddock@facstaff.wisc.edu) 